\title{
ASSESSING URBAN DROUGHTS IN A SMART CITY FRAMEWORK
}

\author{
R. Obringer ${ }^{\text {a }}$, X. Zhang ${ }^{\text {b, c }}$, K. Mallick ${ }^{\text {d }}$, S. H. Alemohammad ${ }^{\text {e }}$, D. Niyogi ${ }^{\text {a, }}{ }^{*}$ \\ ${ }^{a}$ Department Earth, Atmospheric, and Planetary Sciences, Purdue University, West Lafayette, IN, USA - (robringe, \\ dniyogi)@purdue.edu \\ b Department of Agronomy, Purdue University, West Lafayette, IN, USA - zhan2449@purdue.edu \\ ${ }^{\mathrm{c}}$ Key Laboratory of Information Engineering in Surveying, Mapping, and Remote Sensing, Wuhan University, Wuhan 430079, \\ China \\ d Department of ERIN, Luxembourg Institute Science and Technology, L-4422, Belvaux, Luxembourg - kaniska.mallick@list.lu \\ ${ }^{\mathrm{e}}$ Department of Civil and Environmental Engineering, Massachusetts Institute of Technology, Cambridge, MA, USA - \\ hamed_al@mit.edu
}

Theme Session 17

KEY WORDS: Urban Drought, Evapotranspiration, Surface Resistance, Smart City Framework, Water Resiliency, STIC.

\begin{abstract}
:
This study aims to integrate environmental data for drought monitoring to reduce uncertainty in urban drought characterization as part of the smart city framework. Currently, drought monitoring in urban areas is a challenge. This is due, in part, to a lack of knowledge on the subject of urban droughts and urban drought vulnerability. A critical part to assessing urban drought and implementing the necessary policies is determining drought conditions. Often the timing and severity of the drought can leave cities to enforce water restrictions, so accuracy of this determination has socioeconomic implications. To determine drought conditions, we need to know the water balance over the urban landscape, of which evapotranspiration (ET) is a key variable. However, ET data and models have high uncertainty when compared to other hydrological variables (i.e., precipitation). This is largely due to ill-defined empirical models for characterizing the urban surface resistance parameter $\left(r_{s}\right)$ that is used in ET calculations. We propose a method to estimate $r_{s}$ values using a combination of the Surface Temperature Initiated Closure (STIC) method that calculates regional evapotranspiration data and an inverted version of the Penman-Monteith equation. We use this approach across the region surrounding Indianapolis, IN (USA) from 2010-2014. We discuss the potential for this method to be integrated in to smart city framework to improve urban drought assessment.
\end{abstract}

\section{INTRODUCTION}

In the United States, urban drought policies are based off the US Drought Monitor (USDM) classification. For example, when the USDM declares a region to be experiencing a severe drought, a city located in that region may respond by implementing voluntary or mandatory water use restrictions. However, the USDM classification is largely tailored to the surrounding agricultural land, and a city following such a classification could implement restrictions too late, or keep them on too long. By using the USDM classification system alone, a city could be using too much water, thus making the drought worse for surrounding areas, or placing unnecessary restrictions on the citizens, leading to potential economic damages. A solution to this challenge of declaring drought versus non-drought conditions in an urban area could be found within the smart city framework.

Smart cities build on the paradigm of using technology and connectivity to monitor critical infrastructure and improve efficiency (Chourabi et al. 2012). Usually the focus is on the physical state of the infrastructure; however, the framework can also be used to monitor environmental states for hydrological extremes, such as drought, within the city itself.

A city that has implemented environmental monitoring technology would be able to collect and analyse data that would provide insight into their drought vulnerability, and allow for a more customized urban drought policy. Cities would be able to implement restrictions based on the conditions in the city and its watershed, instead of relying solely on the USDM to declare their region to be experiencing a drought. By integrating drought assessment into smart city framework, we expect to be able to improve the robustness of water governance within the city.

Additionally, cities are becoming increasingly large in both area and population. It is predicted that by 2050 , over two-thirds of the world population will be living in cities, most of that growth taking place in the developing world (The World Bank 2010). Meanwhile, the intensive urbanization can lead to changes in the hydro-meteorology of a region, largely due to their ability to change the spatial and temporal patterns of rainfall (Kishtawal et al. 2010; Niyogi et al. 2011). These precipitation changes would likely lead to increased drought vulnerability for the city itself and the surrounding watershed.

As urban populations continue to grow, cities will need to both access and manage increasing volumes of water, which could place more stress on urban watersheds. When a drought occurs, it amplifies this stress and leads to serious societal and economic damages, as seen recently in Los Angeles, USA and San Juan, PR.

Due to the challenges of water governance during drought as well as the increasing role of urbanization on regional hydro-climates, it is imperative that we improve the mode through which we

\footnotetext{
Corresponding author
} 
assess urban droughts. The smart city framework offers a rich opportunity to not only improve drought assessment, but also positively impact the lives of the people living in cities.

That being said, drought assessment in cities is a difficult task, due to the complex nature of urban climate and the unique socioeconomic characteristics of cities. This study seeks to improve the calculation of evapotranspiration (ET) and surface resistance over urban areas.

ET is a central hydrological input to any drought assessment technique, however, urban ET calculations often have inaccuracies that stem from parameterized biophysical state variables in the widely-used Penman-Monteith (PM) equation, specifically, the surface resistance parameter.

This study uses a method that overcomes the parameterization uncertainties in the PM equation and directly estimates ET. This gridded ET is more regionally representative than traditional calculations and can be used in drought vulnerability assessment. Although this study only focuses on testing the accuracy of the model and determining the viability for future urban drought research, it is our goal to implement the method in a smart city framework to determine drought vulnerability in real-time.

\section{METHODS}

The premise of this study as well as future research, is that if we know surface resistance over urban areas as a function of the Local Climate Zone (LCZ), then we will be able to import that surface resistance data into models, such as WRF or Noah, and retrieve accurate ET values. This will in turn help to improve urban drought vulnerability assessments and characterization. In order to get to the point where we are running models with improved surface resistance values, we need to first find what these urban surface resistance values are and how they can be attributed to LCZs. The first step was to take a case study and find the surface resistance values based on estimated ET data. For this case study, we chose to study Indianapolis, IN. The following sections provide details on the method we used to find the surface resistance over Indianapolis.

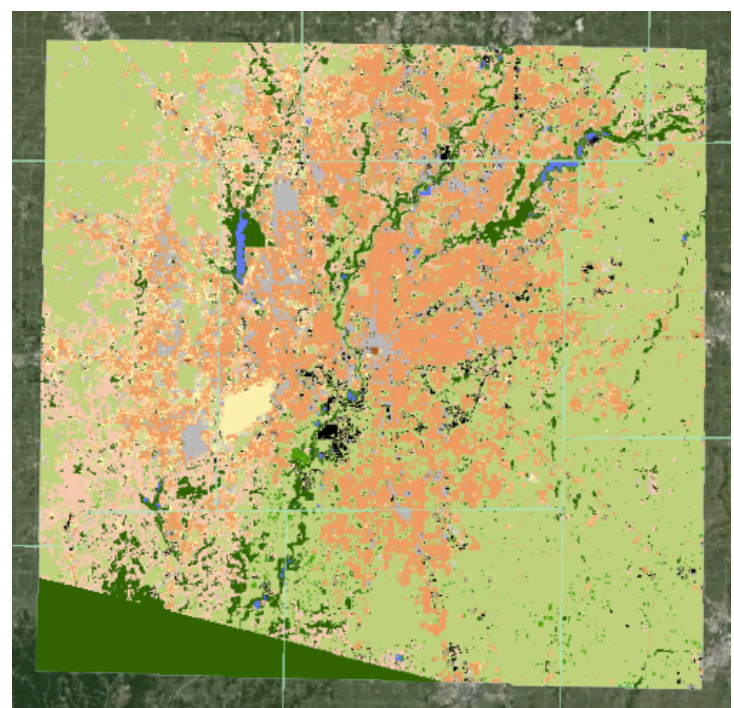

Figure 1: Land use map of Indianapolis and the surrounding area using LCZ framework. The orange represents urban/suburban areas and the green represents rural/forested areas. Map Credit: Oscar Brousse and Anamika Shreevastava, Purdue University.

\subsection{Research Domain}

For the purpose of this study, we chose to work in the Indianapolis region. Indianapolis is the capital city of the state of Indiana located in the Midwest region of the United States. This region encompasses the city proper as well as the surrounding suburban regions. The region, home to nearly two-million people, experienced a variety of hydro-meteorological conditions in recent years - including a record wet year in 2011 and a record dry year in 2012. In order to capture these two extremes, we chose to study the evapotranspiration over the region from 2010 to 2014. In addition to being known to have experienced a drought in the last five years, Indianapolis is also a city that several authors are familiar with, making it easy to obtain environmental data and city layout information. An LCZ map of Indianapolis can be seen in figure 1 .

\subsection{Data}

The main data source for this study was the NCEP North American Regional Reanalysis (NARR). This reanalysis model uses the NCEP Eta Model in combination with the Regional Data Assimilation System (RDAS). The data is available at daily and monthly temporal scales, and the spatial resolution is 32 kilometres (Mesinger et al. 2003). For urban studies, a finer resolution is ideal, so we chose to use a statistical downscaling approach, which is discussed later. The data collected from NARR was used in two different steps: 1. Input data into STIC; and, 2. Input data into the Penman-Monteith equation. More details on these steps are in the following sections. We chose to use the reanalysis data because it was both complete and accessible, however, the method could be used with in-situ or satellite data.

We also used flux tower data collected by the INFLUX team in Indianapolis. The INFLUX team has several eddy covariance towers around Indianapolis from which they record atmospheric data (Dr. Ken Davis, Penn State University). We used data from January to July 2013 as a control for testing the performance of the STIC method.

\subsection{Implementing the STIC Method}

The Surface Temperature Initiated Closure (STIC) method is a recently-developed technique for calculating ET (Mallick et al. 2014; 2015). This method focuses on finding an analytical solution of the resistances and finding a 'closure' of the PM equation for estimating ET. Inputs to this method include the air and radiometric surface temperatures, relative humidity, net radiation and ground heat flux. As mentioned earlier, this data was obtained from NARR. The output from STIC is a gridded ET dataset. Our input data from NARR was at 32-kilometer gridspacing, therefore our ET dataset had the same. Unfortunately, this resolution is too coarse for the purpose of urban studies. In order to improve this resolution, we used a bilinear interpolation technique to reduce the pixel size to 4 kilometres. This gridspacing, although not ideal, is significantly better than the original resolution. Additionally, we used the monthly mean data from NARR as input to both STIC and the Penman-Monteith equation.

\subsection{Determining the Surface Resistance}

The main contribution of this study was to formulate a new algorithm for finding the surface resistance over urban areas. Traditionally, the surface resistance is determined by using an 
empirical equation that has been parameterized for vegetated land using the "big leaf" assumption. This surface resistance (also called canopy resistance) is a term used in a number of formulas, including the Penman-Monteith equation (eq. 1) when solving for evapotranspiration:

$$
\lambda E=\frac{\Delta\left(R_{\mathbf{n}}-\mathbf{G}\right)+\rho_{\mathbf{a}} \mathbf{c}_{\mathbf{p}} \frac{\mathbf{e}_{\mathbf{s}}-\mathbf{e}_{\mathbf{a}}}{\mathbf{r}_{\mathbf{a}}}}{\Delta+\gamma\left(\mathbf{1}+\frac{\mathbf{r}_{\mathbf{s}}}{r_{\mathbf{a}}}\right)}
$$

Where $r_{a}$ is the aerodynamic resistance $\left(\mathrm{s} \mathrm{m}^{-1}\right), r_{s}$ is the surface resistance $\left(\mathrm{s} \mathrm{m}^{-1}\right), \Delta$ is the slope of the saturation vapour pressure-air temperature curve $\left(\mathrm{kPa}^{\circ} \mathrm{C}^{-1}\right), R_{n}$ is the net radiation $\left(\mathrm{W} \mathrm{m}{ }^{-2}\right), G$ is the ground heat flux $\left(\mathrm{W} \mathrm{m}^{-2}\right), \rho_{a}$ is the air density $\left(\mathrm{kg} \mathrm{m}^{-3}\right), c_{p}$ is the specific heat $\left(\mathrm{J} \mathrm{kg}^{-1}{ }^{\circ} \mathrm{C}^{-1}\right), e_{s}$ is the saturation vapour pressure $(\mathrm{kPa}), e_{a}$ is the actual vapour pressure $(\mathrm{kPa}), \lambda E$ is the ET from the STIC method $\left(\mathrm{W} \mathrm{m}^{-2}\right)$, and $\gamma$ is the psychometric constant $\left(\mathrm{kPa}^{\circ} \mathrm{C}^{-1}\right)$.

Given the ET data obtained from STIC, we were able to invert the Penman-Monteith equation and solve for surface resistance. This inverted Penman-Monteith equation can be stated as:

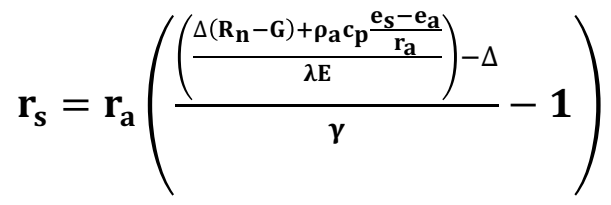

We used the FAO definition of aerodynamic resistance (Allen et al. 1998):

$$
\mathbf{r}_{\mathbf{a}}=\frac{\ln \left(\frac{\mathrm{z}_{\mathrm{m}}-\mathrm{d}}{\mathrm{z}_{\mathrm{om}}}\right) \ln \left(\frac{\mathrm{z}_{\mathrm{h}}-\mathrm{d}}{\mathrm{z}_{\mathrm{oh}}}\right)}{\mathbf{k}^{2} \mathbf{u}_{\mathrm{z}}}
$$

Where $z_{m}$ is the height of the wind measurement $(\mathrm{m}), d$ is the displacement height $(\mathrm{m}), z_{o m}$ is the roughness length governing momentum transfer $(\mathrm{m}), z_{h}$ is the height of the humidity measurement $(\mathrm{m}), z_{\text {oh }}$ is the roughness length governing heat

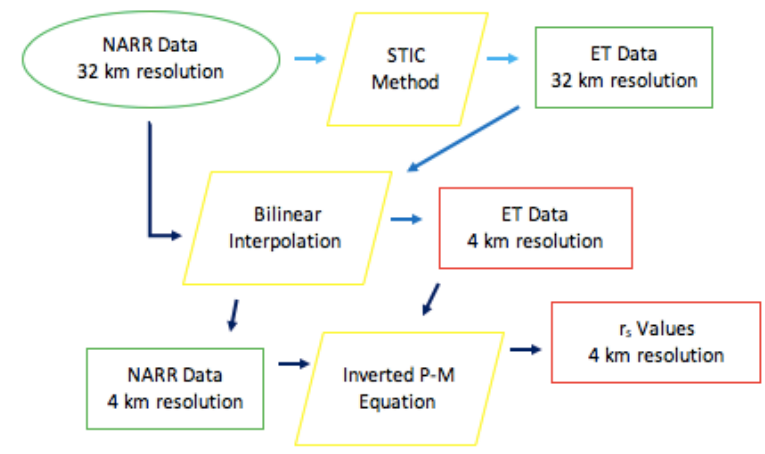

Figure 2: Flow chart depicting the algorithm used to calculate the surface resistance values. The green shapes represent input, the yellow shapes represent processes, and the red shapes represent outputs. Also, the arrows range from light blue to dark blue, each colour representing a new group of steps in the process (dark blue shows the final steps). transfer (m), $k$ is von Karman's constant, and $u_{z}$ is the wind speed $\left(\mathrm{m} \mathrm{s}^{-1}\right)$. It is important to note that for the purpose of this study, we used a displacement height of one meter. The roughness lengths were calculated, again, using the FAO definition.

The process flow for determining the urban surface resistance over Indianapolis is shown in figure 2.

\section{RESULTS}

This study is one of the first in a quest to increase understanding of the effects urbanization has on droughts and the overall hydrological cycle. The purpose was to investigate a new method and determine its accuracy over an urban area. This new approach uses the STIC method to determine the evapotranspiration and then use that data to back-calculate the surface resistance value. Analyses were done to ascertain the spatiotemporal changes in ET and $r_{s}$ as well as the accuracy of the method when compared to flux data. Finally, we analysed the precipitation deficit over time, looking for visual patterns of drought evolution. The following discusses the results of the method and the analyses performed.

\subsection{Evapotranspiration Calculation}

As mentioned earlier, we used the STIC method to find ET over the study domain. We found this method to be fairly accurate, with an $\mathrm{r}^{2}$ value of .84 when compared to the flux tower data (see figure 3). We used the STIC data from a $4 \times 4$ kilometre area surrounding the flux tower site. Given that there is such a strong correlation between observations and the STIC data, we are confident that the method is accurate over urban areas, although we would like to run the method with a higher resolution in the future as part of our ongoing analysis.

As urban areas become larger and more populous, it is important that we understand how they effect the regional climate, especially the hydrological aspect. Our research revolves around the effects on the hydrological cycle, specifically drought in the city and its watershed. Evapotranspiration is an important aspect of the cycle and it can play a major part in determining the drought vulnerability of a city. In figure 4 , we show the changes

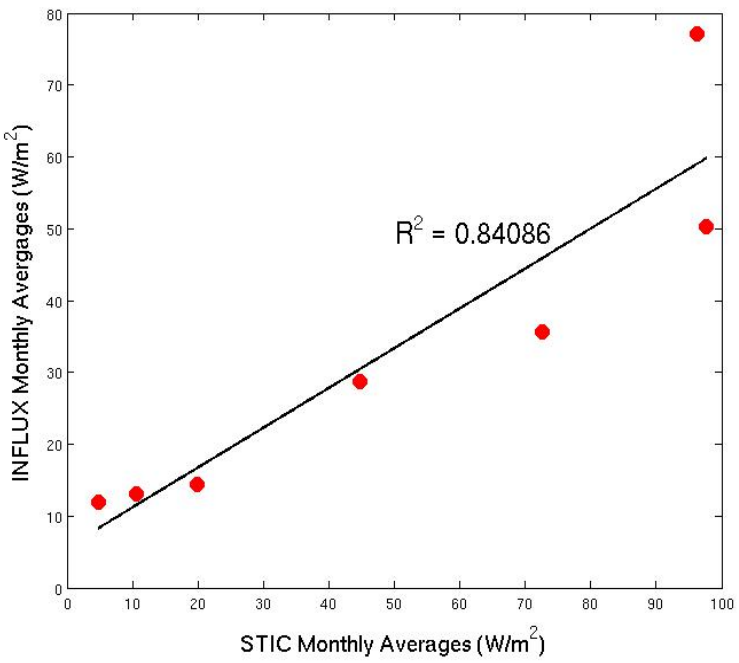

Figure 3: This figure shows the correlation between the ET data collected by INFLUX and the ET data calculated by STIC. The flux tower is located on the West side of Indianapolis, and data was used from January to July 2013. 
in ET over the spring and summer of 2012. In 2012, there was a severe flash drought over much of our research domain. A flash drought is a drought that occurs over a small temporal scale, often for the length of a season. Interestingly, the spring of 2012 was characterized by better-than-normal conditions from a farming

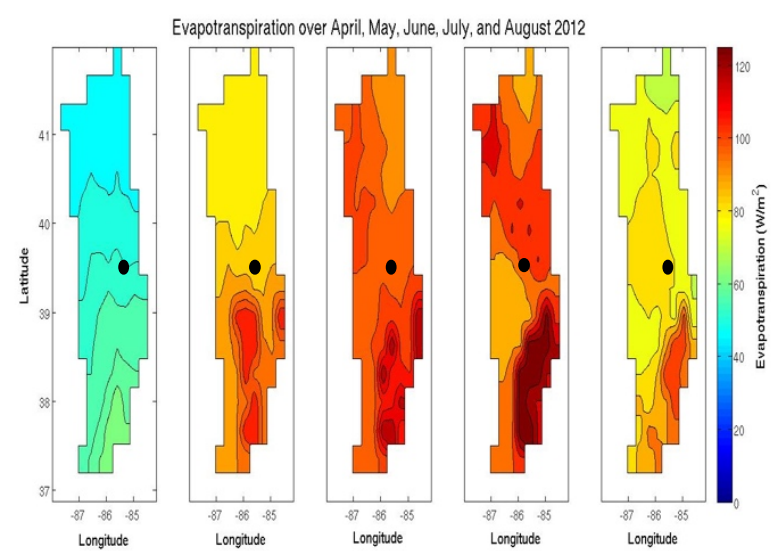

Figure 4: Spatiotemporal changes in ET over the spring and summer of 2012. The black dot represents the location of Indianapolis, IN.
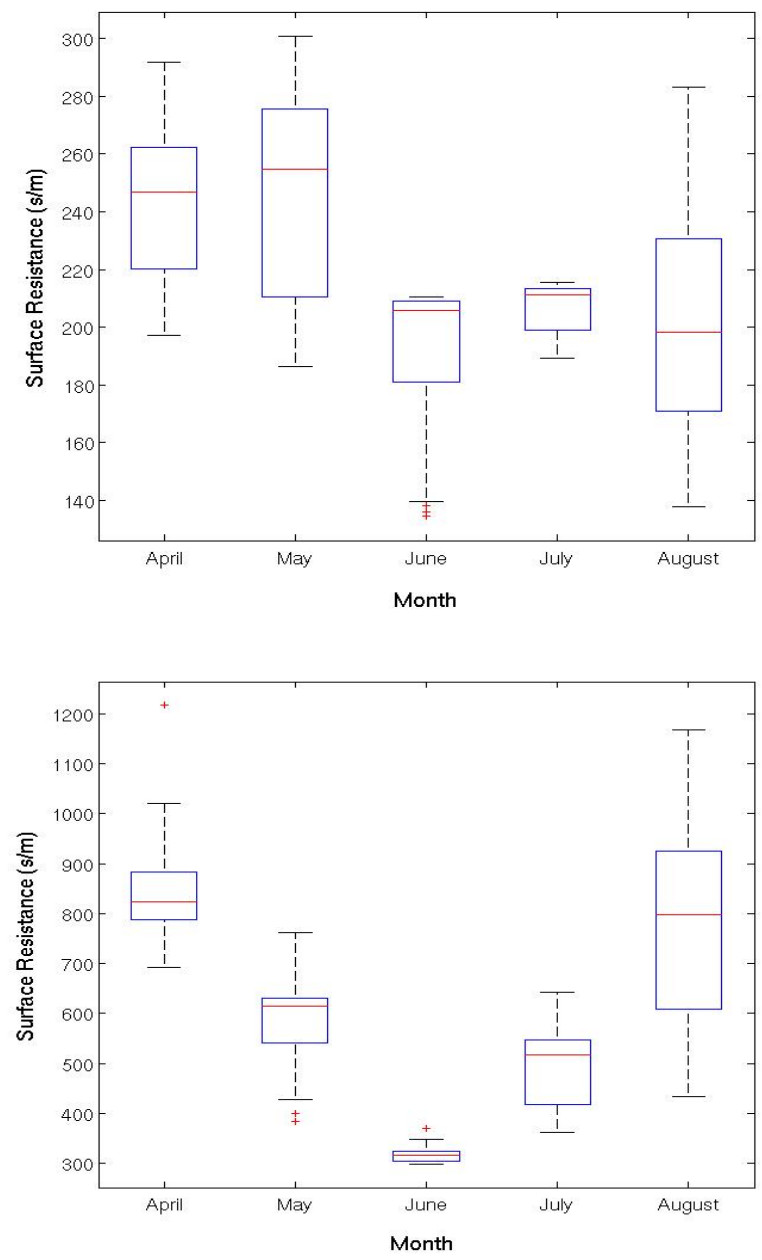

Figure 5: Box plots showing the temporal changes over the spring and summer months of 2011 (upper) and 2012 (lower). Scales are different in the two panels to show the details. perspective, but that quickly changed around May when the entire region entered into a severe drought, as recorded in the USDM. The ET temporal variance (figure 4) shows low ET values in April with a steady increase until July, where ET is maximized in the region. This is an expected seasonal change, but the sharpness of the contrast indicates a need for additional studies, especially since it is known that a severe drought occurred during that time. Further information, such as the precipitation, would be needed to make additional assessment of drought, as shown later in this paper.

It is interesting to note that the area dominated by Indianapolis (denoted by the black dot) is consistently experiencing less ET than neighbouring areas, especially those areas just southeast of the city. This is likely due to the amount of impervious surfaces in the city compared to the amount of pervious surfaces (soils, forests, etc.) throughout much of the area. Without much potential for plant transpiration, it is expected that ET will be lower. Additionally, without precipitation, it is unlikely that there will be any water on the surface to evaporate from the urban area, as there is little irrigation.

The main reason for using the STIC method is to reduce the uncertainties introduced by using a parameterized surface resistance $\left(r_{s}\right)$ value. So by using STIC to find ET, we can then use that ET to back-calculate $r_{s}$. The results of this part of the study are discussed next.

\subsection{Surface Resistance Calculation}

To find the surface resistance over the research domain, we used an inverted Penman-Monteith equation (eq. 2). The surface resistance is often a major source of inaccuracy in ET calculations because it has been parameterized for landscapes with a large amount of plant cover. This leads to uncertainties in urban ET data, which can propagate throughout the models and drought assessments. Our goal is to create an index of urban $r_{s}$ values that are based on building type and vegetative percentage, so that urban model accuracy is increased. Unfortunately, the resolution for the data used in this study was not high enough to perceive any patterns based on LCZs, although efforts are currently underway to get to this point. That being said, the data can still provide insight about the urban surface resistance.

Figure 5 shows the temporal analyses of surface resistance during two contrasting years. The top plot is for 2011, a record wet year in Indiana, while the lower plot is for 2012, a record dry year. During 2011, surface resistance remained fairly constant throughout the spring and summer. But during 2012, the surface resistance was highly variable. Interestingly, the minimum surface resistance in 2012 (June) was higher than then maximum surface resistance in 2011 (May). This suggests that high surface resistance to evapotranspiration may be an indicator of drought conditions in urban areas, however more research needs to be done. It is important to note that for this part of the study, the research domain was just Indianapolis, as opposed to the ET calculations that encompass most of Indiana. This distinction was made because we were interested in seeing the regional effects on ET and the local effects on surface resistance.

As this study is focusing on finding a potential method to improve drought recognition over urban areas, we performed a basic precipitation deficit analysis with our ET data and precipitation data from NARR. 


\subsection{Precipitation Deficit Analysis}

A net precipitation deficit occurs when ET is greater than precipitation, or, in other words, there is more water leaving the surface via ET than is being replaced by precipitation. The Budyko framework uses this fact to determine drought conditions (Budyko 1974). For the purpose of this study we did not use the complete Budyko framework, only the deficit analysis. A deficit was considered when the ET was greater than the precipitation. In order to perform this analysis, we needed to convert the ET from $\frac{W}{\mathrm{~m}^{2}}$ to $\frac{\mathrm{kg}}{\mathrm{m}^{2}}$, which were the units of our precipitation data. The precipitation data was retrieved from NARR as accumulated monthly mean, and we used the bilinear interpolation once again to downscale the data from 32-kilometre to 4-kilometre resolution. The results of this analysis can be seen in figure 6 .

This figure shows the changes in the precipitation deficit over the spring and summer of 2012. The cooler colours represent a deficit in precipitation, while the warmer colours represent a surplus in precipitation. The deficit was at its highest during June and slowly decreased through the summer. This pattern of surplusdeficit-surplus through the season is similar to that of the 2012 surface resistance plot (figure $5 \mathrm{~b}$ ). This suggests that a high precipitation deficit is correlated with lower surface resistance values (and vice versa).
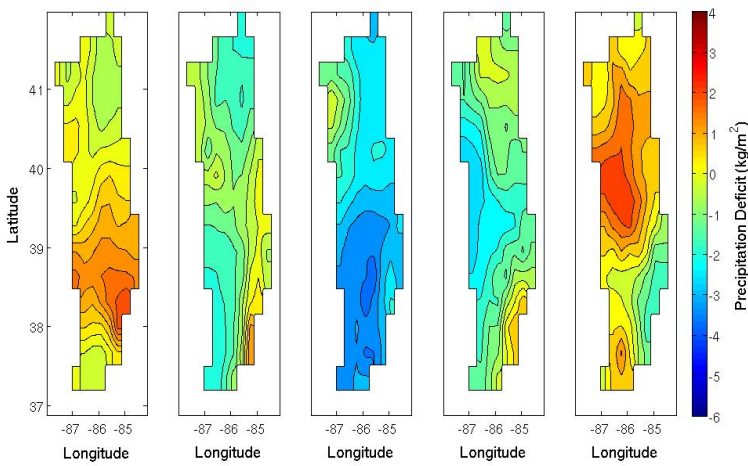

Figure 6: Spatiotemporal changes in precipitation deficit over the spring and summer months of 2012.

\section{CONCLUSIONS}

Overall, this study demonstrated that the STIC method for calculating ET over urban areas is a viable alternative to traditional calculations. Our results show that this method is comparable to urban flux tower data, and therefore encourage its implementation in more areas than the research domain. Our results also show potential correlations between urban drought conditions and urban surface resistance values, although more research is still needed.

Our ultimate goal is to create a method that can be implemented into smart city framework to recognize and assess drought vulnerability. Smart city technology represents an opportunity to link technology with drought monitoring to improve the lives of the citizens. It is our hope that if city water managers have an early warning of drought risk they can plan water policies accordingly. In order to create this method, we need atmospheric data at a much higher resolution than we currently have through NARR. Remote sensing products, such as VIIRS, are a potential source for some of the necessary variables, but not all. For these variables, we need observations at a 100 to 500 -meter resolution. The next steps in our research will be focused on this task.
We are currently looking into two different solutions to the resolution issue. The first is using dynamical downscaling to take the NARR data from 32-kilometer to 500-meter. This process has been demonstrated by Bechtel et al. (2012), and we hope to follow a similar process. The second solution is the Array of Things (AoT) framework that is being implemented in several cities, such as Chicago, USA. This project will get atmospheric and flux data at each street corner in downtown Chicago. We hope to be able to use this data to further investigate the role of ET and surface resistance in predicting and recognizing urban droughts.

The methodology presented is the first step towards improving urban drought recognition and vulnerability assessment. The method is both feasible and accurate to implement in urban areas. Given the data collected and the analyses performed in this study, we are confident that the method outlined can be an alternative to conventional evapotranspiration calculations, which in turn can be a step towards improved urban drought assessment.

\section{ACKNOWLEDGEMENTS}

The authors would like to acknowledge Dr. Ken Davis and his research team for processing and providing the INFLUX data for use in this project. This study benefitted in part by NSF STRONG (CDE 1250232), NSF CAREER (AGS- 847472), and AGS1522494 grants. The authors would also like to acknowledge the support for this research from Purdue University (Andrews Graduate Research Fellowship) as well as the China Scholarship Council (State Scholarship Fund No. 201506270080).

\section{REFERENCES}

Allen, R.G. et al., 1998. Crop evapotranspiration: guidelines for computing crop water requirements. Irrigation and Drainage Paper 56. , p.300.

Bechtel, B., Zakšek, K. \& Hoshyaripour, G., 2012. Downscaling Land Surface Temperature in an Urban Area: A Case Study for Hamburg, Germany. Remote Sensing, 4(10), pp.3184-3200.

Budyko, M.I., 1974. Climate and Life. Academic, San Diego, Calif, pp. $72-191$.

Chourabi, H. et al., 2012. Understanding Smart Cities: An Integrative Framework. 2012 45th Hawaii International Conference on System Sciences, pp.2289-2297.

Kishtawal, C.M. et al., 2010. Urbanization signature in the observed heavy rainfall climatology over India. International Journal of Climatology, 30(13), pp.1908-1916.

Mallick, K. et al., 2014. A Surface Temperature Initiated Closure (STIC) for surface energy balance fluxes. Remote Sensing of Environment, 141, pp.243-261.

Mallick, K. et al., 2015. Reintroducing radiometric surface temperature into the Penman-Monteith formulation. Water Resources research, pp.1-30.

Mesinger, F. et al., 2003. North American Regional Reanalysis: A long-term, consistent, high-resolution climate dataset for the North American domain, as a major improvement upon the earlier global reanalysis datasets in both resolution and accuracy. submitted to BAMS 2004.

Niyogi, D. et al., 2011. Urban modification of thunderstorms: An observational storm climatology and model case study for the Indianapolis urban region. Journal of Applied Meteorology and Climatology, 50(5), pp.1129-1144.

The World Bank, 2010. Cities and Climate Change: An Urgent Agenda, pp.1-92. 